Rev. Psicol. (Arequipa. Univ. Catól. San Pablo) / Año 2021 / Vol 11 / No 1 / pp. 169-187

ISSN 2306-0565 versión impresa / ISSN 2311-7397 versión on line

\title{
Afrontamiento de la Incertidumbre en jóvenes imposibilitados de regresar del extranjero por pandemia de COVID-19
}

\author{
Coping with Uncertainty in Young People Unable to Return \\ from Abroad Due to the COVID-19 Pandemic
}

Gina Chávez-Ventura

Universidad César Vallejo, Trujillo, Perú

(iD) https://orcid.org/oooo-0oo2-4638-3487

Correspondencia: gina.chavezv@hotmail.com

Henry Santa-Cruz-Espinoza

Universidad César Vallejo, Trujillo, Perú

(iD https://orcid.org/oooo-0oo2-6475-9724

César Vásquez-Olcese

Universidad Privada del Norte, Trujillo, Perú

(iD https://orcid.org/oooo-00o2-7232-3250

Janeth Molina-Alvarado

Universidad Privada del Norte, Trujillo, Perú

(iD) https://orcid.org/oooo-0oo2-6451-8613

\section{Resumen}

Para evitar la propagación de la COVID-19, muchos países —entre ellos Perúcerraron sus fronteras, imposibilitando el retorno de peruanos que tenían previsto volver al país y ya no contaban con recursos económicos para su manutención al haber concluido sus contratos laborales. Por ello, el propósito del estudio es caracterizar los modos de afrontamiento a la incertidumbre que experimentaron jóvenes peruanos cuando se encontraban imposibilitados de regresar a su país debido a la pandemia por COVID-19. Se diseñó un cuestionario con preguntas abiertas y cerradas, se obtuvo la validez de contenido $(V=1[.90,1])$ y se aplicó, mediante formularios Google, a 39 jóvenes entre 19 y 26 años $(M=20.9, D E=1.6)$, que cumplían su cuarentena, tras ser repatriados por el gobierno peruano. Los resultados reportan que los jóvenes se sintieron especialmente agobiados o angustiados (64.10\%) y tensos (56.41\%). Sus principales temores eran no poder regresar a Perú (43.8\%) y 
contagiarse de la COVID-19 (25\%). Afrontaban el malestar durmiendo (22.81\%), realizando actividades físicas para relajarse (21.03\%), recibiendo apoyo emocional de personas que recién conocían (79.49\%), y también proporcionaron apoyo a otras personas (80.95\%). Casi la totalidad de los participantes (92.31\%) realizaron acciones para intentar solucionar el problema, y el $100 \%$ buscaba estar informado de las noticias. La mayoría (74.36 \%) valoró como excitante la experiencia vivida de estar imposibilitados de poder retornar a su país. Se concluye que las principales acciones realizadas han sido para afrontar la incertidumbre emocional, cognitiva $y$ de deseo de cambio.

Palabras clave: Tolerancia a la ambigüedad, incertidumbre, afrontamiento, emociones.

\begin{abstract}
To prevent the spread of COVID-19, many countries, including Peru, closed their borders. As result, it was impossible for Peruvians to return to their country although they no longer had the financial resources to support themselves due to the ending of their employment contracts. The purpose of this study is to describe the ways of coping with uncertainty utilized by young Peruvians while they were unable to return to their country due to the COVID-19 pandemic. A questionnaire with open and closed questions was designed, content validity was obtained ( $V=$ $1[.90,1])$ and it was applied, using Google forms, to 39 young people between 19 and 26 years of age $(M=20.9, S D=1.6)$. The sample was surveyed during their quarantine period, following their repatriation by the Peruvian government. The results indicated that the young people felt especially overwhelmed or distressed (64.10 \%) and tense (56.41\%) prior to returning to Peru. Their main fears were not being able to return to Peru (43.8\%) and becoming infected with COVID$19(25 \%)$. They coped with discomfort by sleeping (22.81\%), by doing physical activities to relax (21.03\%), by receiving emotional support from people they just met (79.49\%), and, also by providing support to others (80.95\%). Almost all of the participants (92.31\%) carried out actions to try to solve the problem; $100 \%$ wanted to be informed of the COVID-19 news. The majority (74.36\%) valued the experience of being unable to return to their country as an exciting experience. This study concluded that the main actions taken were undertaken to deal with emotional, cognitive uncertainty and a result of a desire for change.
\end{abstract}

Keywords: Tolerance of ambiguity, uncertainty, coping, emotions.

\section{Introducción}

La pandemia ocasionada por la enfermedad COVID-19 ha generado, a nivel mundial, un impacto de gran magnitud a nivel económico, político, psicosocial y sanitario (Babore et al. 2020; Dawson \& Golijani-Moghaddam, 2020; Ramírez et al., 2020), razón por la cual diversos gobiernos han decretado una serie de 
disposiciones para contener la propagación rápida de la enfermedad infecciosa; entre ellas el aislamiento social y el cierre de fronteras. Tal es el caso del Estado Peruano que, en comparación a los demás países latinoamericanos, decretó tempranamente (17 de marzo de 2020) la prohibición de ingreso y salida del país, así como los viajes interprovinciales, entre otras medidas.

Ello dio lugar a que tanto los extranjeros, que estaban temporalmente en el Perú, como los peruanos en el extranjero viesen frustrados sus planes de retorno, sin tener claro cuál sería el plan de contingencia ni cuándo se resolvería su situación. Dentro del último grupo, más de 3000 corresponden a jóvenes del programa de intercambio cultural y laboral Work and Travel, que no pudieron retornar de EE.UU. (El Comercio, 19 de marzo de 2020).

Participan de dicho programa estudiantes universitarios que viajaron a Estados Unidos, por su período vacacional de diciembre a marzo, realizan trabajos remunerados (que les permiten su subsistencia), practican el inglés y se relacionan con estudiantes de otras nacionalidades. Sin embargo, el cierre de fronteras representaba para ellos: a) la pérdida de su pasaje aéreo de retorno al país sin saber si lo recuperarían, b) el vencimiento de su visa J-1 por trabajo temporal, c) la caducidad de su seguro de salud que cubría el período laboral, d) la caducidad de contrato laboral, e) tener, en varios casos, que desalojar el lugar de residencia temporal por el cese de trabajo, f) tener que cubrir sus gastos de hospedaje y manutención por un tiempo indeterminado sin tener ingresos económicos actuales, y g) estar lejos de su hogar. A esto se suma el riesgo de estar expuestos a un posible contagio de la COVID-19, la falta de una política de contención del gobierno estadounidense, las cuarentenas impuestas por los consulados, así como la incertidumbre de no saber la fecha de reapertura de los vuelos internacionales o desconocer si se será favorecido o no, con los vuelos de repatriación promovidos por el gobierno peruano en coordinación con los consulados. Todo ello representa una gran incertidumbre con respecto a su situación y su porvenir.

El fenómeno de la incertidumbre ha sido investigado especialmente desde el campo biomédico, en el cuidado de la salud (Brabow \& Kline, 200o) y más ampliamente vinculado a la enfermedad (Mishel, 1988; Wiener \& Dodd, 1993; Yang et al., 2015). Desde este último, la teoría explica que la valoración de la incertidumbre hacia la enfermedad puede ser positiva o negativa (Mishel, 1990). En el primer caso, la incertidumbre se considera como una oportunidad y en el segundo como un peligro, ya que las situaciones imprevistas irrumpen el sentido de control y planificación de la vida del ser humano, así como su homeostasis (Mishel, 1990), incluso a nivel hormonal (Voigt, Ziegler, Grunert-Fuchs, Bicke \& Fehm-Wolfsdorf, 1990) e inmune (Zakowski, 1985).

La valoración negativa de la incertidumbre se ve impulsada especialmente por la cultura con una visión mecanicista de la vida, donde se valora la precisión, 
la autoeficacia y el control interno. Por tanto, la incertidumbre se constituye en un potencial perturbador de las áreas del desenvolvimiento humano asociado a la angustia psicológica (Mishel, 1990), representa preocupación, inmoviliza para actuar, se valora como negativa e injusta (Buhr \& Dugas, 2002); y, su intolerancia actúa como mediadora entre el neuroticismo, la depresión y ansiedad (Clarke \& Kiropoulos, 2021).

En cambio, en el campo de la educación, la incertidumbre se percibe como una de las características de las sociedades del conocimiento (García-Cortés, 2019) y se considera equiparable a la gestión del riesgo que exige enfrentar el estrés y la ambigüedad (Walker et al., 2017). También se utiliza como una estrategia didáctica en los proyectos formativos, orientados al desarrollo del pensamiento complejo para la resolución de problemas del contexto con un producto real (Tobón et al., 2015).

En el presente estudio, el interés por comprender el afrontamiento a la incertidumbre se enfocó desde el ámbito psicosocial, específicamente en cómo los jóvenes peruanos hacen frente el no saber cómo resolver la situación de estar imposibilitados de regresar a su país, por el cierre de fronteras debido a la pandemia por COVID-19. Ante el carácter novedoso y coyuntural del fenómeno a investigar, no se encontraron instrumentos disponibles que puedan medir la variable de interés, debido a que los estudios instrumentales más próximos están enfocados en el afrontamiento al estrés (Lyne \& Roger, 2000; Tobin et al., 1989). Sin embargo, un reporte mostró la construcción de una medida de las vivencias emocionales de la incertidumbre (Greco \& Roger, 2001), en lugar de las estrategias empleadas para afrontar la experiencia emocional que genera la incertidumbre.

Para el estudio se asumen dos teorías, la que explica el afrontamiento al estrés y la que teoriza las respuestas a la incertidumbre. La primera se realiza desde el modelo transaccional del estrés de Lazarus y Folkman (1984), que afirma la interacción del individuo con el medio ambiente, sobre el cual puede ejercer influencia a través de estrategias conductuales, cognitivas o emocionales; sin embargo, cuando se presenta una discrepancia entre las demandas del ambiente y los recursos para hacerles frente, surge el estrés.

Ante ello, los esfuerzos cognitivos y conductuales para intentar manejar tales demandas, que exceden los recursos del individuo, y que procuran reducir el impacto emocional o alterar el problema, se denominan afrontamiento; el cual varía en su uso y duración y puede expresarse en tres estrategias: a) las orientadas al problema (e.g. conductas dirigidas a buscar información y prepararse activamente para estar en condiciones de afrontar el problema); b) las orientadas a la emoción (e.g. conductas orientadas a requerir el soporte de personas, con el fin de solucionar el problema o desahogar la emoción); y, c) las de evitación (e.g. conductas de postergación o evasión) (Lazarus \& Folkman, 1984). 
Vale señalar que la teoría transaccional relaciona las estrategias de afrontamiento con las emociones, luego de que se valora el suceso estresante, $y$ forma una unidad con el afrontamiento (Lazarus, 1999); por lo que es necesario reconocer la presencia de emociones positivas y negativas, su intensidad y duración (Berra et al., 2014). Las emociones positivas contribuyen al bienestar y las negativas al malestar (Padrós et al., 2012). También, es necesario conocer a quién se atribuye la responsabilidad del evento, si el suceso estresante afectó la consecución de las metas y cómo se valoraron las consecuencias, a nivel de la relación con los demás y el ambiente (Berra et al., 2014); aunque, se critica la falta de claridad y precisión respecto a las emociones negativas y estrés, en las formulaciones de Lazarus (Gómez, 2005).

En la segunda teoría, Greco y Roger (2001) señalan que la incertidumbre es un estilo de respuesta desadaptativo, que comprende tres tipos de respuesta. La incertidumbre emocional (incluye las respuestas fisiológicas y psicológicas a la anticipación de amenaza) se relaciona positivamente con el neuroticismo, está fuertemente relacionada con el estrés y rumiación y negativamente relacionada con la autoestima y el desapego. La incertidumbre cognitiva (desequilibra la necesidad de planificación y certeza) se relaciona inversamente con la tolerancia a la ambigüedad y marginalmente con la sensibilidad social del neuroticismo; mientras que el deseo de cambio (disfrute de incertidumbre, novedad y cambio) se relaciona con la impulsividad y sociabilidad e incluye la búsqueda de sensaciones.

Cabe señalar que, si una persona logra tolerar la incertidumbre, es capaz de tolerar la angustia del otro y contribuir a reducir de modo prosocial su malestar; por lo que el afrontamiento de la incertidumbre está mediado por habilidades de autorregulación y la habilidad cognitiva de asumir una perspectiva (Saarni et al., 2007).

Para el estudio, se asumen las definiciones siguientes: a) afrontamiento a la incertidumbre es el conjunto de esfuerzos cognitivos y conductuales para manejar la situación de desconcierto y ambigüedad por estar varados en un país extranjero; b) afrontamiento a la incertidumbre emocional, comprende conductas de búsqueda de apoyo o soporte social, para solucionar el problema o reducir el impacto afectivo mediante el desahogo de la emoción; c) afrontamiento a la incertidumbre cognitiva, abarca conductas de búsqueda de información y de preparación activa para estar en condiciones de afrontar el problema; y d) afrontamiento del deseo de cambio, que implica conductas que buscan mantener la excitación ante lo novedoso.

Por lo expuesto, el estudio responde a la necesidad de caracterizar los modos de afrontamiento a la incertidumbre que experimentaron jóvenes peruanos imposibilitados de regresar a su país por el cierre de fronteras debido a la 
pandemia de la COVID-19. Con ello, se posibilitará ampliar las investigaciones sobre el afrontamiento a la incertidumbre, desde un contexto no vinculado a la enfermedad, y comprender las vivencias emocionales de quienes han tenido que afrontar la incertidumbre de no saber cuándo retornarían al país en situaciones adversas para ellos.

\section{Método}

El diseño es de tipo selectivo, descriptivo, con muestreo no probabilístico transversal (Ato et al., 2013).

\section{Participantes}

Formaron parte del estudio jóvenes que viajaron al extranjero y estuvieron imposibilitados de regresar por el cierre de fronteras debido a la pandemia de la COVID-19. Los participantes se encontraban haciendo cuarentena en los hoteles limeños, designados por el gobierno peruano, tras ser repatriados o recientemente habían retornado a sus hogares luego del confinamiento obligatorio dispuesto por el Estado.
El estudio se llevó a cabo entre los meses de abrilajuniode 2020, en el momentoenqueel gobierno estadounidense no había dispuesto medidas de cuarentena, sino que algunos Estados los asumían voluntariamente y también los Consulados peruanos al asignar hoteles financiados por el gobierno peruano, para peruanos que estuvieran confinados.

Entre las características de los participantes, la mayoría son mujeres, y tienen edades entre 19 y 26 años $(\mathrm{M}=20.9, \mathrm{DE}=$ 1.6). El $100 \%$ son solteros, el $89.74 \%$ no presentan problemas de salud, el 94.87 $\%(\mathrm{n}=35)$ vive con su familia y proceden en su mayoría de la capital. Además, tras determinar el cierre de fronteras, los participantes se quedaron en casa de un familiar, o de amigos, o en el lugar de trabajo, o en el aeropuerto, y posteriormente en un hotel dispuesto por el Consulado peruano (Tabla 1).

La cantidad de días que estuvieron en el extranjero sin poder regresar era desde $2 \mathrm{a}$ 65 días $(M=25.51, D E=15.78)$ y el tiempo transcurrido desde que retornaron a Perú es desde 1 día hasta 62 días $(M=39.18$, $\mathrm{DE}=18.43$ ) 
Tabla 1.

Características de los participantes

\begin{tabular}{|c|c|c|}
\hline Características & f & $\%$ \\
\hline \multicolumn{3}{|l|}{ Sexo } \\
\hline Mujer & 23 & 59 \\
\hline Hombre & 16 & 41 \\
\hline \multicolumn{3}{|l|}{ Procedencia } \\
\hline Lima & 23 & 58.97 \\
\hline Trujillo & 9 & 23.08 \\
\hline Arequipa & 3 & 7.69 \\
\hline Huancayo & 2 & 5.13 \\
\hline Chiclayo & 1 & 2.56 \\
\hline Ilo & 1 & 2.56 \\
\hline \multicolumn{3}{|l|}{ Carreras profesionales } \\
\hline Ingeniería & 15 & 38.46 \\
\hline Ciencias Administrativas & 7 & $17 \cdot 95$ \\
\hline Comunicaciones & 6 & $15 \cdot 38$ \\
\hline Salud & 4 & 10.26 \\
\hline Traducción & 2 & 5.13 \\
\hline Arte & 2 & 5.13 \\
\hline Humanidades & 3 & 7.69 \\
\hline \multicolumn{3}{|l|}{ Dónde se quedó tras declararse el cierre de fronteras: } \\
\hline Casa de familiar o amigo & 8 & 20.51 \\
\hline Lugar de trabajo & 13 & 33.33 \\
\hline Aeropuerto y luego hotel dispuesto por Consulado & 12 & 30.77 \\
\hline Lugar de trabajo y luego hotel dispuesto por & 3 & 7.69 \\
\hline Consulado & 3 & 7.69 \\
\hline Hotel pagado por mí & & \\
\hline \multicolumn{3}{|l|}{ Durante ese tiempo se encontró: } \\
\hline Solo & 5 & 12.82 \\
\hline Acompañado de amigos & 30 & 76.92 \\
\hline Acompañado de familiares & 4 & 10.26 \\
\hline
\end{tabular}

\section{Instrumento}

Debido a la imposibilidad de acceder a la población objetivo se aplicó, vía Internet, una ficha de datos sociodemográficos y un cuestionario (Tabla 2), diseñados para los fines de la investigación, con 10 preguntas abiertas y 11 cerradas. Para la obtención de la validez basada en el contenido, seis psicólogos evaluaron de manera positiva el instrumento, con una escala de 7 puntos, los criterios de claridad, relevancia y pertinencia $(\mathrm{V}=1$ $[.90,1])$. Además, se incluyó un rubro de observaciones para que puedan sugerir modificaciones al instrumento. 
Tabla 2.

Preguntas del Cuestionario sobre Afrontamiento a la Incertidumbre

\author{
Afrontamiento a 1. De las siguientes emociones indica, ¿cuáles describen mejor cómo te sentías \\ la incertidumbre durante el tiempo que tuviste que quedarte en el extranjero sin poder regresar a \\ emocional Perú? Puedes elegir más de una alternativa. \\ 2. ¿Qué temores tenías? Menciónalos \\ 3. ¿Qué acciones o qué tipo de conducta realizabas cuando las emociones o \\ sentimientos te generaban malestar? Menciónalas todas \\ 4. Durante el tiempo que estuviste en el extranjero sin poder regresar al Perú, \\ ¿preferías estar solo o acompañado? \\ 5. ¿Qué cambios experimentaste en tu organismo, durante ese tiempo? \\ 10. ¿Recibiste el apoyo emocional de personas importantes para ti? Marca \\ quiénes fueron. \\ 11. ¿Recibiste apoyo emocional de personas que recién conocías? \\ 12. Si tu respuesta fue "Sî" a la pregunta anterior, ¿de qué manera te apoyaron? \\ 13. ¿Brindaste apoyo a otras personas? \\ 14. Si tu respuesta fue "SÎ" a la pregunta anterior, ¿de qué manera brindaste \\ apoyo?
}

Afrontamiento a la incertidumbre cognitiva
6. ¿Intentaste algún modo de resolver la situación en la que te encontrabas para volver a Perú?

7. Si tu respuesta fue "SÎ" a la pregunta anterior, ¿qué fue lo que hiciste?

8. ¿Quiénes contribuyeron a encontrar la solución para tu pronto retorno al país?

9. De acuerdo tu respuesta a la pregunta anterior, explica qué fue lo que hicieron

15. Durante ese tiempo, ¿con qué frecuencia te informabas por las noticias, de la situación de los peruanos en el extranjero y las medidas para su retorno al Perú? (ejemplo: disposiciones del gobierno, comunicaciones del Consulado por la web)

18. ¿Procuraste planificar y cumplir una rutina diaria?

19. ¿Con qué frecuencia organizabas tu equipaje para retornar al Perú?

Afrontamiento a

16. Consideras que la experiencia de estar en el extranjero sin poder regresar al

la incertidumbre

del deseo de cambio
17. Si tu respuesta a la pregunta anterior fue "SI", explica por qué

20. Mientras estuviste en el extranjero sin poder retornar al país, ¿te refugiaste en alguna religión, credo o doctrina filosófica?, explica cómo

21. Cuando estuviste en el extranjero sin poder retornar al país, ¿encontraste algo positivo en esa experiencia vivida? explica 


\section{Procedimiento}

Diseñado el cuestionario, los jueces procedieron a evaluar los ítems de manera independiente, vía correo electrónico. Luego de tener la versión final de la prueba, se realizó laselección de los participantescon un muestreo no probabilístico por conveniencia.

Primero, se solicitó la autorización de una agencia de Work and Travel, la misma que envío los enlaces virtuales a los estudiantes para que, quienes deseen, participen del estudio. Además, algunos participantes contactaron a sus conocidos con la técnica de bola de nieve. En segundo lugar, para garantizar el bienestar y la autonomía de los participantes de estudio, se solicitó su consentimiento informado, vía formularios Googlea través de WhatsApp, previa presentación del equipodeinvestigación, el objetivo del estudio, el carácter libre y voluntario de la participación, así como el anonimato de sus respuestas. De ese modo, se garantizaron los principios éticos en investigación científica para los participantes (American Psychological Association, 2016; Colegio de Psicólogos del Perú, 2017). Quienes estaban de acuerdo en participar, seleccionaron la opción "sí" y continuaron con el llenado del formulario, en cambio, quienes elegían la alternativa "no", daban por finalizada su participación.

\section{Análisis de datos}

Para la cuantificación de la valoración de los especialistas en la validez de contenido del instrumento aplicado se utilizó la V de Aiken y sus intervalos de confianza (Penfield \& Giacobbi, 2004), mientras que para el análisis descriptivo de la información recolectada se efectuó mediante las frecuencias relativas simples y porcentuales.

\section{Resultados}

\section{Afrontamiento a la incertidumbre emocional}

Antes de presentar los hallazgos acerca de cómo los participantes afrontaron la incertidumbre emocional cuando estuvieron en el extranjero imposibilitados de retornar al Perú, se explicará las emociones que hicieron frente. Experimentaron en mayor proporción emociones negativas, debido a que se sintieron agobiados o angustiados (64.10 $\%)$, tensos (56.41 \%), nerviosos (41.03 $\%)$, intranquilos (41.03\%) y temerosos (41.03\%). Vivenciaron dichas emociones tanto quienes estuvieron pocos días en el extranjero antes de regresar a Perú, como quienes estuvieron mayor tiempo. Sin embargo, los que permanecieron de 45 a 65 días en el exterior sin poder retornar experimentaron más frecuentemente la coexistencia de emociones negativas y positivas. Vale decir, que la presencia de estas últimas emociones se presentó también en quienes tuvieron menos tiempo en el exterior, destacando como emociones positivas, sentirse esperanzado y activo (Tabla 3 ). 
Tabla 3.

Emociones experimentadas durante el tiempo en el extranjero sin poder regresar a Perú

\begin{tabular}{|c|c|c|c|c|c|}
\hline \multirow{2}{*}{$\begin{array}{l}\text { Días sin poder } \\
\quad \text { regresar }\end{array}$} & \multirow{2}{*}{\multicolumn{2}{|c|}{ Emociones }} & \multirow{2}{*}{$f$} & \multicolumn{2}{|c|}{ Total } \\
\hline & & & & $f$ & $\%$ \\
\hline \multirow[t]{7}{*}{$1-14$} & Negativas & Agobiado o angustiado, irritado & 3 & & \\
\hline & & Agobiado o angustiado, temeroso, tenso & 1 & 5 & 50 \\
\hline & & Temeroso, tenso, intranquilo & 1 & & \\
\hline & Ambas & Tenso, decidido, esperanzado & 1 & & \\
\hline & & Enojado, nervioso, esperanzado & 1 & & \\
\hline & & Agobiado, temeroso, esperanzado & 2 & 5 & 50 \\
\hline & & $\begin{array}{l}\text { Dispuesto, temeroso, nervioso, atento, } \\
\text { activo }\end{array}$ & 1 & & \\
\hline \multirow[t]{8}{*}{$15-29$} & Negativas & Agobiado, tenso, temeroso & 9 & & \\
\hline & & Agobiado o angustiado, enérgico & 1 & 10 & 62.5 \\
\hline & Positivas & Decidido, atento, activo, esperanzado & 1 & & \\
\hline & & Dispuesto, decidido, activo & 1 & 3 & 18.75 \\
\hline & & Animado, enérgico, atento & 1 & & \\
\hline & Ambas & Agobiado, tenso, esperanzado & 1 & & \\
\hline & & Tenso, entusiasmado, intranquilo, asustado & 1 & 3 & 18.75 \\
\hline & & Tenso, orgulloso, nervioso, inspirado & 1 & & \\
\hline \multirow[t]{6}{*}{$30-44$} & Negativas & Agobiado o angustiado, temeroso, tenso & 1 & & \\
\hline & & Temeroso, tenso, intranquilo & 1 & & \\
\hline & & Enojado, irritado, tenso & 1 & 4 & 57.14 \\
\hline & & Agobiado o angustiado & 1 & & \\
\hline & Positivas & Esperanzado & 1 & 1 & 14.29 \\
\hline & Ambos & $\begin{array}{l}\text { Agobiado o angustiado, enojado, } \\
\text { esperanzado }\end{array}$ & 2 & 2 & 28.57 \\
\hline \multirow[t]{6}{*}{$45-65$} & Negativas & Agobiado o angustiado, tenso & 1 & 1 & 16.67 \\
\hline & Ambos & Agobiado o angustiado, activo, esperanzado & 1 & & \\
\hline & & $\begin{array}{l}\text { Agobiado o angustiado, animado, tenso, } \\
\text { inspirado }\end{array}$ & 1 & & \\
\hline & & $\begin{array}{l}\text { Tenso, atento, intranquilo, activo, } \\
\text { esperanzado }\end{array}$ & 1 & 5 & 83.33 \\
\hline & & Agobiado o angustiado, tenso, orgulloso & 1 & & \\
\hline & & Agobiado o angustiado, temeroso, irritado & 1 & & \\
\hline Total & & & & 39 & 100 \\
\hline
\end{tabular}

Los participantes vivenciaron más de un temor a la vez y los más frecuentes fueron no poder regresar a Perú ( $43.8 \%)$, contagiarse de la COVID-19 (25\%), quedarse sin dinero $(12.5 \%)$ y preocupación por sus padres o temor a que se contagien (8.4\%). Además, entre los cambios en el organismo, los participantes refirieron experimentar especialmente alteraciones en el sueño (23.47\%), tensión muscular 
(22.45\%), cambios en el apetito (15.31\%), inquietud (14.29\%), problemas respiratorios o estomacales o dolor físico (16.32 $\%)$, alergias (4.08\%); y, fueron muy pocos quienes refirieron no vivenciar cambio alguno (4.08\%).

El $98 \%$ de los jóvenes accionaron modos de afronte ante las emociones displacenteras experimentadas por la incertidumbre, siendo las más frecuentes: dormir (22.81 $\%)$, intentar relajarse efectuando actividades físicas (21.03\%), realizar acciones de distracción como jugar o ver televisión o series (17.54\%), hablar con la familia o amigos (12.28\%), llorar (12.28\%), escuchar música (7.02 \%), salir con amigos (3.51 \%), mientras que una persona no experimentó emociones displacenteras $(1.75 \%)$ y otra se autolesionaba.

En relación al apoyo emocional, éste lo ha proporcionado la familia (64.23\%), los amigos (22.76 \%) y la pareja (11.38\%). Vale señalar que el 79.49\% prefería estar acompañado que solo. Asimismo, una gran cantidad de los jóvenes participantes refirieron haber recibido apoyo emocional de personas que recién conocían (79.49 $\%)$; y, también la mayoría de los participantes $(80.95 \%)$ proporcionó apoyo a otras personas, de tipo emocional $(59.52$ $\%)$, material (14.29\%) o con información (7.14\%).

\section{Afrontamiento a la incertidumbre cognitiva}

Casi la totalidad de los participantes (92.31\%) realizaron acciones para intentar solucionar el problema, entre las cuales destacaron: contactarse con el Consulado (43.59\%), contactar con aerolíneas y vuelos chárter $(25.64 \%)$, organizarse en grupos para viaje (5.13\%), contactar con las agencias de Work and Travel (5.13 $\%)$, buscar ayuda de otros peruanos en igual situación (5.13\%) y contactarse con medios de comunicación (5.13\%).

Los que contribuyeron en la solución del problema, según la percepción de los participantes, fueron el Consulado peruano (40.35\%), los familiares (29.82 $\%)$, amigos (19.30 \%) y desconocidos (10.53 $\%)$. El tipo de apoyo recibido fue verse favorecido con el vuelo de retorno $(52.63$ $\%)$, haberse comunicado con los medios que posibiliten la solución del problema (39.47\%), recibir apoyo emocional ( 18.42 $\%)$, hospedaje y alimentación (5.26 \%) y apoyo económico (2.63\%).

El 100\% de los participantes buscaba estar informado de las noticias sobre la situación de los peruanos en el extranjero y las disposiciones de Estado para su retorno al Perú. La mayoría se actualizaba en las noticias diariamente (74.36\%), y otros lo hacían con menor frecuencia, como interdiaria $(15.38 \%)$, semanal (7.69 \%) y mensual (2.56\%). Además, el 53.85\% procuró cumplir una rutina diaria y organizaba su equipaje con cierta regularidad (28.21 \% semanal, $12.82 \%$ diaria y 12.82 $\%$ interdiaria).

\section{Afrontamiento del deseo de cambio}

Los participantes valoraron en su mayoría $(74.36 \%)$ como excitante o emocionante la experiencia vivida de 
estar imposibilitados de poder retornar a su país. Calificaron la situación como una experiencia nueva $(38.46 \%)$, de supervivencia o aventura (15.38\%), o que favoreció la autorregulación personal o la maduración $(10.26 \%)$ y les permitió conocer personas y solidarizarse $(2.56$ $\%)$. Solamente el $33.33 \%$ calificó la experiencia como "mala". Además, el 92\% de los participantes no se apoyó en alguna religión alguna, ni credo, ni doctrina filosófica mientras estuvo en el extranjero sin poder regresar al Perú.

Finalmente, ante la pregunta de si hubo algo positivo en la experiencia vivida, los participantes respondieron que aprendieron a controlar sus emociones 34.78 $\%)$, se hicieron más independientes o maduros $(26.09 \%)$, les ayudó a valorar a su familia (13.04\%), les permitió tener amigos (21.74\%) y sólo una persona respondió que el evento acentuó la depresión $(4.35 \%)$.

\section{Discusión}

El estudio se efectuó con el propósito de caracterizar los modos de afrontamiento a la incertidumbre de jóvenes peruanos que estuvieron imposibilitados de retornar a su país por el cierre de fronteras debido a las disposiciones del gobierno peruano por la pandemia de la COVID-19. Vale señalar que los jóvenes participantes son estudiantes universitarios, participantes del programa Work and Travel, quienes en su mayoría viven con su familia y tras declararse el cierre de fronteras tuvieron que permanecer en EE.UU., acompañados principalmente por amigos.
Los participantes, mientras no podrían regresar del extranjero, mayoritariamente vivenciaron la incertidumbre con emociones negativas (angustia, tensión, nerviosismo, intranquilidad y temor). Al respecto, se concuerda con un estudio que vincula los síntomas ansiosos respecto a la COVID-19 con la dificultad para tolerar la incertidumbre (Wheaton et al., 2021). Sin embargo, los que tenían mayor tiempo experimentaron en mayor porcentaje la coexistencia de emociones negativas y positivas, entre estas últimas se contaban la esperanza y sentirse activo, las mismas que son necesarias para orientar las estrategias para alcanzar las metas (Castillo et al., 2014).

Entre los temores, el más frecuente fue el riesgo de no poder regresar al Perú, seguido por el miedo a contagiarse de la COVID-19 y es que estar expuestos a las noticias - por necesidad- en el contexto de la pandemia resulta estresante (Vásquez et al., 2020); y más aún, ello se agudiza si se suma el no disponer de un seguro de salud debido a la finalización del contrato laboral y estar lejos de sus familias. Además, se adicionaron alteraciones en el sueño, así como dolencias físicas, que están asociadas al estrés (Del Río Portilla, 2006).

A pesar de ello, lo que contribuyó a que los jóvenes participantes manejen la situación de desconcierto y ambigüedad y a que reduzcan el impacto afectivo de lo vivido, fue haber estado acompañado, en su mayoría por amigos, haber recibido el apoyo emocional de la familia en la distancia, incluso el soporte de desconocidos, y 
haber practicadoactividades de ocio, lo que favorece la salud mental (Goodman et al., 2017). Posiblemente ello explique la puesta en marcha de acciones de apoyo en favor de quienes vivían igual experiencia, que les ayudó a tolerar su propia incertidumbre y a disminuir la que enfrentaba el otro, favoreciendo la autorregulación emocional (Saarni et al., 2007), la misma que fue señalada por los participantes como experiencia positiva, al haber madurado y aprendido a controlar las propias emociones.

Aún con la emocionalidad vivida, los participantes - durante su periodo vacacional-, obtuvieron la experiencia de haber dirigido su vida por sí mismos y haber trabajado, en un contexto cultural diferente y lejos a su entorno familiar, donde establecieron nuevos vínculos amicales. Ello posiblemente puede haber contribuido a que afronten la incertidumbre cognitiva activamente, buscando información y los recursos para intentar encontrar solución a su situación, lo que corresponde a la estrategia centrada en el problema, según Lazarus y Folkman (1984).

Aunque la solución final de la situación no dependió de ellos, el resultado no se hubiese alcanzado sin la puesta en marcha de las gestiones realizadas, que solamente benefició a algunos jóvenes bajo la misma situación. Por ello, la atribución acerca de quien contribuyó en la solución del problema fue dirigida especialmente al Consulado peruano, seguido por familiares y desconocidos. Además, el cumplimiento de una rutina diaria, seguida por la mayoría de los jóvenes, implicaba que se organicen y ejerzan el control sobre la distribución de su tiempo, lo que favorece el equilibrio y contribuye a la calidad de vida (Gómez, 2006), en un contexto de incertidumbre en el porvenir.

Respecto al afrontamiento de la incertidumbre del deseo de cambio, los participantes en su mayoría, experimentaron lo vivido como excitante o emocionante, lo que podría corresponder con el hallazgo de que la búsqueda de sensaciones y novedades se incrementa hacia la adolescencia y disminuye en la adultez, ya que la muestra evaluada fue en su mayoría de jóvenes con un promedio de 20 años (Alcázar et al., 2015). La solución a su situación con vuelos humanitarios o chárter, no dependía de los jóvenes, por lo que prolongar o no la situación de incertidumbre, no estaba bajo su control; sin embargo, las experiencias vividas contribuyeron a su autorregulación emocional, de acuerdo a lo referido por los participantes.

Cabe señalar que, como limitaciones del estudio, se considera haberlo realizado con una reducida cantidad de participantes, integrada únicamente por jóvenes peruanos del programa Work and Travel, lo que afecta la validez externa de la investigación. Además, no fue posible acceder a muestras con un tiempo mayor de permanencia en el extranjero ( $>65$ días) ni a quienes aún se encontraban en el extranjero sin poder retornar; por lo que las experiencias reveladas por los participantes fueron con una percepción en retrospectiva, cuando ya habían sido repatriados, acerca de cómo afrontaron la situación mientras aún permanecían 
en el exterior. Estudios futuros podrían superar estas limitaciones, para posibilitar una mayor comprensión de la variable de estudio, porque gran parte de los antecedentes se han focalizado al estudio de la incertidumbre vinculado a la enfermedad.

Otra de las limitaciones ha sido el diseño transversal del estudio, que imposibilitó recoger información sobre los cambios ocurridos a nivel fisiológico, emocional, cognitivo, comportamental y el afrontamiento de los participantes, desde el inicio hasta el término de su situación de incertidumbre. Asimismo, haber recogido información únicamente con una medida de autorreporte y no combinarlas con medidas físicas fue otra limitación del estudio.

Al no haber tenido disponible un instrumento estandarizado que evalúe el constructo de interés, como parte del estudio, se diseñó un cuestionario que incluye preguntas abiertas para recoger mayor profundidad en las respuestas. Para éste, sólo se obtuvo una evidencia de validez basada en el contenido, que constituye un aporte para comprender el constructo desde el ámbito psicosocial, poco explorado.

Aunque se recolectó información sobre el afrontamiento a la incertidumbre, en los hallazgos se reportan aspectos comunes con el afrontamiento al estrés, en la mayoría de los participantes, quienes se sintieron agobiados o tensos, como respuesta a la coyuntura política y sanitaria que impedía el regreso a su país, activándose en ellos respuestas fisiológicas, cognitivas y comportamentales, al percibir que se desbordan sus recursos personales ante una situación que está fuera de su control y que afecta su bienestar; y que se encaminó fundamentalmente al empleo de estrategias de centradas en el problema y en la emoción (Lazarus \& Folkman, 1984).

Sin embargo, como señaló Lazarus, el factor tiempo es un elemento que se debe considerar en el estudio del afrontamiento al estrés, y, en el caso del presente estudio, la cantidad promedio de días bajo incertidumbre vividas por los jóvenes fue de 25; por lo que no todos los participantes experimentaron estrés, pero sí todos vivenciaron la incertidumbre de su porvenir en un contexto de pandemia. Además, la perspectiva asumida para el estudio, permitió obtener, entre la información del afrontamiento a la incertidumbre emocional, el proveer de apoyo a otros, y no solo recibirlo. Asimismo, se exploró el afrontamiento a la incertidumbre de deseo de cambio, expresado en la excitabilidad y novedad de la experiencia vivida. Estudios futuros podrían utilizar medidas cuantitativas de intolerencia a la incertidumbre (Buhr \& Dugas, 2002), estilos de afrontamiento y considerar otras variables como la flexibilidad cognitiva, porque comprende la disposición o resistencia al cambio (Dennis \& Vander Wal, 2010).

Se concluye que los jóvenes peruanos del programa Work and Travel, que estuvieron imposibilitados de regresar a su país por el cierre de fronteras, debido a la pandemia por la COVID-19, utilizaron 
modos de afrontamiento a la incerti- afrontamiento de la incertidumbre desde dumbre emocional, expresado en temo- un panorama distinto al de la enfermedad res, emocionalidad negativa y malestar y en un contexto sin precedentes, como físico, con el soporte de amigos, familia- lo es la pandemia por la COVID-19. res y proveer apoyo a otros. El afrontamiento a la incertidumbre cognitiva, fue buscando alternativas de solución para su retorno a Perú, informándose de las noticias y cumpliendo rutinas diarias; mientras que, por el afrontamiento de deseo de cambio, la experiencia vivida se percibió como una experiencia excitante. Finalmente, esta información Los autores manifiestan no tener conflicto permitió caracterizar el fenómeno del de interés alguno. 


\section{Referencias}

Alcázar, M. A., Verdejo, A., Bouso, J. C., \& Ortega, J. (2015). Búsqueda de sensaciones y conducta antisocial. Anuario de Psicología Jurídica, 25, 75-8o. http://dx.doi. org/10.1016/j.apj.2015.01.003

American Psychological Association (2016). Ethical Principles of Psychologists and Code of Conduct. https://www.apa.org/ethics/code

Arias, M. M., \& Giraldo, C. V. (2011). El rigor en la investigación cualitativa. Investigación y Educación en Enfermería, 29(3), 500-514. https://www.redalyc. org/pdf/1052/105222406020.pdf

Ato, M., López, J. J., \& Benavente, A. (2013). Un sistema de clasificación de los diseños de investigación en Psicología. Anales de Psicología, 29(3), 1038-1059. http:// dx.doi.org/10.6018/analesps.29.3.178511

Babore, A., Lombardi, L., Vicenconti, M. L., Pignataro, S., Marino, V., Crudele, M., ..., Trumello, C. (2020). Psychological effects of the COVID-2019 pandemic: Perceived stress and coping strategies among healthcare professionals. Psychiatry Research, 293. https://doi.org/10.1016/j.psychres.2020.113366

Berra, E., Muñoz, S., Vega, C., Silva, A. \& Gómez, G. (2014). Emociones, estrés y afrontamiento en adolescentes desde el modelo de Lazarus y Folkman. Revista Intercontinental de Psicología y Educación, 16(1), 37-57. https://www.redalyc. org/pdf/802/80230114003.pdf

Brabow, A., \& Kline, K. (200o). From "reducing" to "coping with" uncertainty: reconceptualizing the central challenge in breast self-exams. Social Science $\mathcal{E}$ Medicine, 51(12), 1805-1816. https://doi.org/10.1016/So277-9536(oo)oo112-X

Buhr, K., \& Dugas, M. J. (2002). The intolerance of uncertainty scale: psychometric properties of the English version. Behaviour Research and Therapy, 40(8), 931-945. https://doi.org/10.1016/Sooo5-7967(o1)ooog2-4

Castillo, H., Urrutia, C., Shimabukuro, M., \& Caycho, T. (2014). Análisis psicométrico del índice de esperanza de Herth en una muestra no clínica peruana. Psicología desde el Caribe, 31(2). http://dx.doi.org/10.14482/psdc.31.2.5974

Clarke, E., \& Kiropoulos, L. (2021). Mediating the relationship between neuroticism and depressive, anxiety and eating disorder symptoms: The role of intolerance 
of uncertainty and cognitive flexibility. Journal of Affective Disorders Reports, 4. https://doi.org/10.1016/j.jadr.2021.100101

Colegio de psicólogos del Perú (2017). Código de ética y deontología. CPP.

Dawson, D., \& Golijani-Moghaddam, N. (2020). COVID-19: Psychological flexibility, coping, mental health, and wellbeing in the UK during the pandemic. Journal of Contextual Behavioral Science, 17(20), 126-134. https://doi.org/10.1016/j. jcbs.2020.07.010

Del Río Portilla, I. (2006). Estrés y sueño. Revista Mexicana de Neurociencia, 7(1), 15-20. http://previous.revmexneurociencia.com/wp-content/uploads/2014/o6/ Nmo61-03.pdf

Dennis, J. P., \& Vander Wal, J. S. (2010). The Cognitive Flexibility Inventory: instrument development and estimates of reliability and validity. Cognitive Therapy and Research, 34, 241-253. https://doi.org/10.1007/s106o8-009-9276-4

El Comercio (19 de marzo de 2020). Work and Travel asegura que 3.0oo estudiantes peruanos de su programa están varados en EE.UU. https://elcomercio.pe/mundo/ eeuu/coronavirus-en-estados-unidos-work-and-travel-30oo-peruanos-estan-varados-en-diferentes-lugares-del-pais-covid-19-pandemia-nndc-noticia/?ref=ecr

García-Cortés, G. (2019). Modelo integral de calidad para las universidades politécnicas de México. Un enfoque desde la socioformación. Atenas, 3(47), 70-89. https:// atenas.reduniv.edu.cu/index.php/atenas/article/view/49o/761

Gómez, S. (2006). Equilibrio y organización de la rutina diaria. Revista Chilena de Terapia Ocupacional, 6. https://doi.org/10.5354/o719-5346.2010.111

Gómez, V. (2005). Richard Stanley Lazarus (1922-2002). Revista Latinoamericana de Psicología, 37(1), 207-209. https://www.redalyc.org/pdf/805/80537112.pdf

Goodman, W. K., Geiger, A. M., \& Wolf, J. M. (2017). leisure activities are linked to mental health benefits by providing time structure: comparing employed, unemployed and homemakers. Journal of Epidemiology and Community Health, 71(1), 4-11. http://dx.doi.org/10.1136/jech-2016-20726o

Greco, V. \& Roger, D. (2001). Coping with uncertainty: the construction and validation of a new measure. Personality and Individual Differences, 31, 519-534. 
Lazarus, R. S. (1999). Stress and emotion. A new synthesis. Springer.

Lazarus, R. S., \& Folkman, S. (1984). Stress appraisal and coping. Springer.

Lyne, K., \& Roger, D. (200o). A psychometric re-assessment of the COPE questionnaire. Journal of Personality and Individual Differences, 29(2), 321-335. https:// doi.org/10.1016/So191-8869(99)oo196-8

Mishel, M. H. (1988). Uncertainty in Illness. Image: The Journal of Nursing Scholarship, 20(4), 225-232. https://doi.org/10.1111/j.1547-5069.1988.tbooo82.x

Mishel, M. H. (1990). Reconceptualization of the Uncertainty in Illness Theory. Image: The Journal of Nursing Scholarship, 22(4), 256-262. https://doi. org/10.1111/j.1547-5069.1990.tboo225.x

Padrós, F., Soriano-Mas, C., \& Navarro, G. (2012). Afecto positivo y negativo: ¿Una dimensión bipolar o dos dimensiones unipolares independientes? Interdisciplinaria, 29(1), 151-164.

Penfield, R. D., \& Giacobbi, P. R. (2004). Applying a Score Confidence Interval to Aiken's Item Content-Relevance Index. Measurement in Physical Education and Exercise Science, 8(4), 213-225. https://doi.org/10.1207/s15327841mpeeo804_3

Ramírez, F. B., Misol, R. C., Alonso, M. del C. F., \& Tizón, J. L. (2020). Pandemia de la Covid-19 y Salud Mental: reflexiones iniciales desde la atención primaria de salud española. Atención Primaria. https://doi.org/10.1016/j.aprim.2020.06.0o6

Saarni, C., Campos, J. J., Camras, L. A., \& Witherington, D. (2007). Emotional Development: Action, Communication, and Understanding. Handbook of Child Psychology. https://doi.org/10.1002/9780470147658.chpsyo305

Tobin, D. L., Holroyd, K. A., Reynolds, R. V., \& Wigal, J. (1989). The hierarchical factor structure of the coping strategies inventory. Cognitive Therapy and Research, 13, 343-361. https://doi.org/10.1007/BFo1173478

Tobón, S., Cardona, S., Vélez, J., \& López, J. (2015). Proyectos formativos y desarrollo del talento humano para la sociedad del conocimiento. Acción Pedagógica, 24, 20-31.

Yang, D., Ye, J., Zhou, F., Li, J., Huang, Q., \& Wan, L. (2015). Relationship between uncertainty in illness, mood state and coping style in patients with temporomandibular 
disorders. International Journal of Nursing Sciences, 2(4), 361-365. https://doi. org/10.1016/j.ijnss.2015.11.003

Vásquez, G., Urtecho-Osorto, O., Agüero-Flores, M., Díaz-Martínez, M., Paguada, R., Varela, M., Landa-Blanco, M., \& Echenique, Y. (2020). Salud mental, confinamiento y preocupación por el coronavirus: un estudio cualitativo. Revista Interamericana de Psicología, 54(2), e1333.

Voigt, K., Ziegler, M., Grunert-Fuchs, M., Bickel, U., \& Fehm-Wolfsdorf, G. (1990). Hormonal responses to exhausting physical exercise: the role of predictability and controllability of the situation. Psychoneuroendocrinology, 15, 173-184. https:// doi.org/10.1016/0306-4530(90)90028-8

Walker, D., Davis, P., \& Stevenson, A. (2017). Coping with uncertainty and ambiguity through team collaboration in infrastructure projects. International Journal of Project Management, 35(2), 180-19o. https://doi.org/10.1016/j.ijproman.2016.11.001

Wheaton, M., Messner, G., \& Marks, J. (2021). Intolerance of uncertainty as a factor linking obsessive-compulsive symptoms, health anxiety and concerns about the spread of the novel coronavirus (COVID-19) in the United States. Journal of Obsessive-Compulsive and Related Disorders, 28. https://doi.org/10.1016/j. jocrd.2020.100605

Wiener, C., \& Dodd, M. (1993). Coping amid uncertainty: an illness trajectory perspective. Scholarly inquiry for nursing practice: An International Journal, 7(1), 17-31. https://www.researchgate.net/profile/Carolyn_Wiener/publication/14711661_Coping_Amid_Uncertainty_An_Illness_Trajectory_Perspective/ links/555a647co8ae6943a877ce46/Coping-Amid-Uncertainty-An-IllnessTrajectory-Perspective.pdf

Zakowski, S. G. (1985). The effects of stressor predictability on lymphocyte proliferation in humans. Psychology and Health, 10, 409-425. https://doi. org/10.108o/o887044950840196o

Recibido: 22 de febrero de 2021

Revisado: 2 de abril de 2021

Aceptado: 7 de mayo de 2021 